\title{
The influence of image reconstruction on two-dimensional spectrograms of the solar photosphere
}

\author{
K. Mikurda ${ }^{1}$, A. Tritschler ${ }^{2}$, and W. Schmidt ${ }^{1}$ \\ 1 Kiepenheuer-Institut für Sonnenphysik, Schöneckstr. 6, 79104 Freiburg, Germany \\ e-mail: [kasia; wolfgang]@kis.uni-freiburg.de \\ 2 National Solar Observatory/Sacramento Peak Observatory, PO Box 62, Sunspot, NM 88349-0062, USA \\ e-mail: ali@nso.edu
}

Received 14 June 2005 / Accepted 28 March 2006

\section{ABSTRACT}

\begin{abstract}
Aims. We present a spectral analysis of small-scale structures in the solar photosphere and investigate the influence of the speckle deconvolution technique on the line profiles.

Methods. A short sequence of two-dimensional spectra is used, taken with the Telecentric Etalon Solar Spectrometer (TESOS) at the German Vacuum Tower Telescope on Tenerife. We observed two small pores surrounded by disturbed and by regular granulation in the non-magnetic neutral Iron line at $557.6 \mathrm{~nm}$. In a first step, a speckle reconstruction is computed by applying an extended KnoxThompson algorithm to the broad-band data. In a second step, the individual narrow-band filtergrams are deconvolved utilizing the information gained in the first step. We then perform a spectral analysis of the 2D spectra and compare the results obtained with the raw and the restored data.

Results. Important spectral quantities, e.g. line position, line depression and line asymmetry are largely unchanged by the image reconstruction process. We derive the line asymmetry and the line-of-sight flow for granules and intergranular lanes and also for an isolated G-band bright point and find important differences between quiet and magnetically disturbed granulation: the granule centers in the quiet region show a strong asymmetry with significant blue shift $(300 \mathrm{~m} / \mathrm{s})$ toward deeper layers, while the velocity in the disturbed area show virtually no height dependence. For the intergranular lanes the situation is reversed: no height dependence in the quiet area, significant red-shift toward deeper layers in the disturbed part. An isolated G-band bright point does not show any lineof-sight motion relative to its immediate surroundings. The map of LOS velocities derived from line-wing shifts shows a significant downflow around one of the pores measured in deep layers of the photosphere.

Conclusions. In most cases we do not find any artefacts in the reconstructed line profiles that would compromise their usage for quantitative spectroscopy.
\end{abstract}

Key words. Sun: photosphere - techniques: spectroscopic - Sun: granulation

\section{Introduction}

The spatial resolution of solar observations has been greatly improved in recent years, due to the availability and the use of real-time image reconstruction techniques. To date, several solar telescopes are equipped with adaptive optics systems (e.g. von der Lühe et al. 1989, 2003; Scharmer et al. 2003; Keller et al. 2003; Rimmele et al. 2004). In addition, image reconstruction techniques can be applied to further improve the effective spatial resolution and image quality. Post-facto techniques are based on reconstruction of either the wavefront or the point-spread-function, and the object scene. During the past decades, basically three approaches have been followed: (a) speckle methods (e.g. Knox \& Thompson 1974; von der Lühe 1993; Weigelt 1977; de Boer \& Kneer 1992), (b) phase-diversity techniques (e.g. Gonsalves \& Chidlaw 1979; Paxman \& Fienup 1988; Löfdahl \& Scharmer 1994), and (c) deconvolution techniques (for a review see Starck et al. 2002). Recent developments of image reconstruction algorithms combine different approaches and apply the methods to observations already supported by adaptive optics (Löfdahl \& Scharmer 2003; Rouppe van der Voort et al. 2004).

Most applications of the aforementioned techniques concentrate on the reconstruction of imaging data. Narrow-band data, i.e. filtergrams with a bandwidth of $5 \mathrm{pm}$ or less, taken at high spatial resolution, do not have sufficiently high signal-to-noise ratios to allow for a direct application of the image reconstruction procedures. A few attempts to combine image reconstruction techniques and one-dimensional slit spectroscopy exist (e.g. Keller \& Johannesson 1995; Sütterlin \& Wiehr 2000). Speckle techniques have been used to restore 2D spectra obtained from filtergraph observations by using the speckle technique and performing a subsequent deconvolution to the narrow-band filtergrams (e.g. Krieg et al. 1999; Hirzberger \& Kneer 2001).

We apply an image reconstruction procedure that is similar to the one described by Krieg et al. (1999), except that our data has already been improved in real time by an adaptive optics system. We measure the line bisectors to derive the line asymmetry and line-of-sight velocity at different heights for all structures in our field-of-view. We use the 2D data to select individual granule centers, dark intergranular lanes and bright points and investigate the contributions of the immediate surroundings to the line profile, its shift and asymmetry. Based on this information, we investigate the influence of the image reconstruction procedure on the line profiles.

The paper is organized as follows. In Sects. 2 and 3 the observations are described and the calibrating and pre-processing 


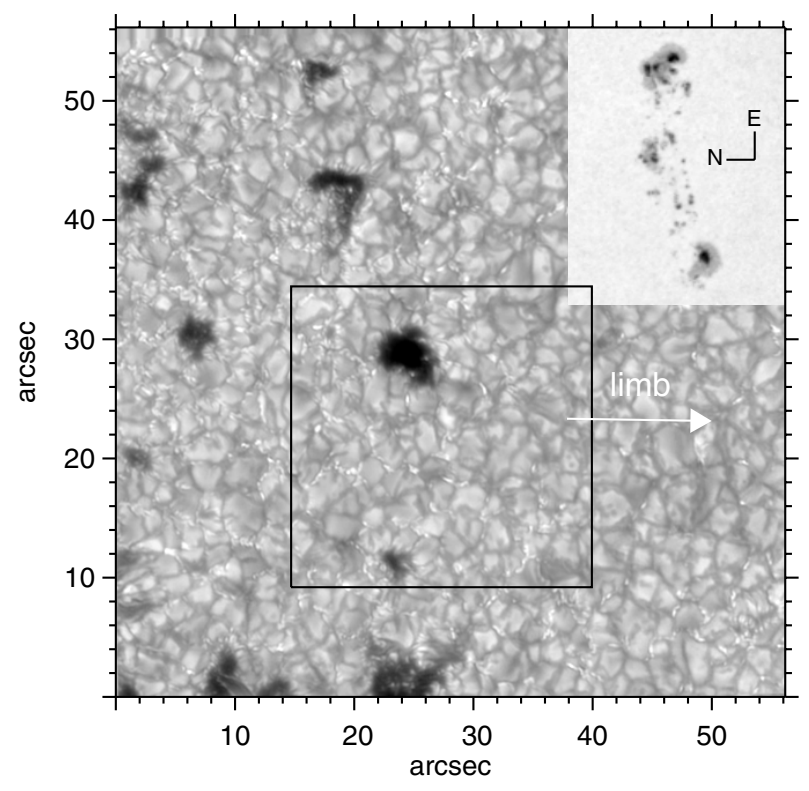

Fig. 1. Speckle reconstructed G-band image. The square indicates the TESOS FOV. The arrow points toward the nearest solar limb. The inset in the upper right corner shows the complete active region AR 10180.

steps are summarized. Section 4 gives a brief overview of the applied image reconstruction techniques. In Sect. 5 we present the results of the spectral analysis and compare the results based on the unrestored data with those of the reconstructed data set. In Sect. 6 we summarize the scientific results.

\section{Observations}

Observations were taken on November 7th, 2002 at the German Vacuum Tower Telescope on Tenerife, Canary Islands using the two-dimensional Fabry-Perot Interferometer TESOS (Telecentric Etalon SOlar Spectrometer, Kentischer et al. 1998; Tritschler et al. 2002) and the adaptive optics system (von der Lühe et al. 2003). TESOS had a circular field-ofview (FOV) of 40 arcsec in diameter and a spectral resolution, $\lambda / \Delta \lambda$, of 250000 . The data set used for this analysis consists of five scans taken in the non-splitting $g=0$ neutral Iron line at $557.6 \mathrm{~nm}$ covering $250 \mathrm{~s}$ of two small pores surrounded by granulation in the active region AR 10180 located at $\theta=15.68^{\circ}$ $(\mu=0.96)$ on the southern hemisphere. The line was sampled at 76 wavelength points with equidistant steps of $0.84 \mathrm{pm}$. The pre-filter had a $F W H M$ of $1.1 \mathrm{~nm}$ and a maximum transmission of $70 \%$. The exposure time and the cycle time were $10 \mathrm{~ms}$ and $30 \mathrm{~s}$, respectively. The broad-band channel of TESOS recorded simultaneously data in a $10 \mathrm{~nm}$ wide $(F W H M)$ wavelength band centered around $550 \mathrm{~nm}$.

The two-dimensional spectroscopic data was complemented by G-band observations performed at the same time with an optical setup placed in front of the TESOS entrance field stop. A dichroic beam splitter plate and a relay optics fed the light beam onto the $1024 \times 1024$ pixel detector of a fast Dalsa camera. The dichroic beam splitter plate reflects any light $<450 \mathrm{~nm}$. A $1.0 \mathrm{~nm}$ wide interference filter centered around $430.3 \mathrm{~nm}$ located in front of the detector was used to select the $\mathrm{G}$ band. The pixel scale was $0.055 \times 0.055 \operatorname{arcsec}^{2}$ and the FOV was $57 \times 57 \operatorname{arcsec}^{2}$. The G-band data was taken in bursts of 100 images each to allow for speckle reconstruction. Figure 1 shows the speckle reconstructed G-band FOV. The TESOS FOV is indicated by the black square. AR 10180 taken from a MDI continuum image is displayed in the upper right corner.

The observations took advantage of the Kiepenheuer Adaptive Optics System (KAOS, von der Lühe et al. 2003).

\section{Data reduction}

Pre-processing procedures involve dark subtraction and gain correction at each individual wavelength step. The flat field images are taken by moving the secondary coelostat mirror during exposure. Slight differences in orientation and pixel image scale between the narrow-band and the broad-band channel of TESOS are determined from the comparison of grid target images. The broad-band images are then corrected for a residual rotation and image scale differences to match the narrow-band images. Residual image motion during the scan is compensated by determining the image shifts from one broad-band image to the next and shifting the simultaneous narrow-band images by the same amount. The intensity modulation pattern (e.g. Tritschler et al. 2002; Tritschler et al. 2004) is removed by Fourier filtering leaving a residual variation on a $1-2 \%$ level in the local continuum intensity. The G-band images have a different orientation and pixel image scale than the TESOS data. In order to identify locations of G-band bright points in the TESOS data, co-spatiality of the two data sets must be guaranteed. Therefore, a residual rotation was applied to the G-band data and its pixel scale was changed.

\section{Image restoration}

As already mentioned, speckle reconstruction techniques are widely used to improve the spatial resolution of filtergrams. However, the transmission band typical for 2D spectroscopic data is much narrower, resulting in a smaller number of photons and a lower signal-to-noise ratio (SNR). Since exposure times required by speckle imaging are of the order of $10 \mathrm{~ms}$, and pixel sizes are usually small in order to achieve the desired spatial resolution, the number of photons would not be sufficient to apply one of those techniques.

The solution proposed by Keller \& von der Lühe (1992) is to take simultaneous broad-band filtergrams and narrow-band images close-by in wavelength and perform speckle imaging of broad-band channel data only. Combining the reconstruction and single broad-band images gives information on the instantaneous state of the Earth's atmosphere. This information is then used to correct the single narrow-band images.

The wavelength sampling, the number of images per wavelength position and the effective camera integration time determine the total time to take a single $2 \mathrm{D}$ spectrum. This time interval should not exceed 30-40 s, otherwise - especially at high spatial resolution - the observed structures in the photosphere may change during the wavelength scan. In addition, a cadence of $40 \mathrm{~s}$ is needed to properly sample the solar oscillations. In practice, a trade-off between wavelength sampling and SNR, i.e. number of images per wavelength position has to be made.

Keller \& von der Lühe (1992) took 100 images at single wavelength in about $30 \mathrm{~s}$. In order to keep the scanning time short, Krieg et al. (1999) took 5 images at each of the 28 wavelength positions chosen to scan the $\mathrm{NaD}_{2}$ spectral line with Fabry-Perot interferometer. Hirzberger \& Kneer (2001) scanned Fe I line $557.6 \mathrm{~nm}$ at 11 wavelength positions with 10 frames per wavelength step, which results in a cadence of subsequent scans of $70 \mathrm{~s}$. 

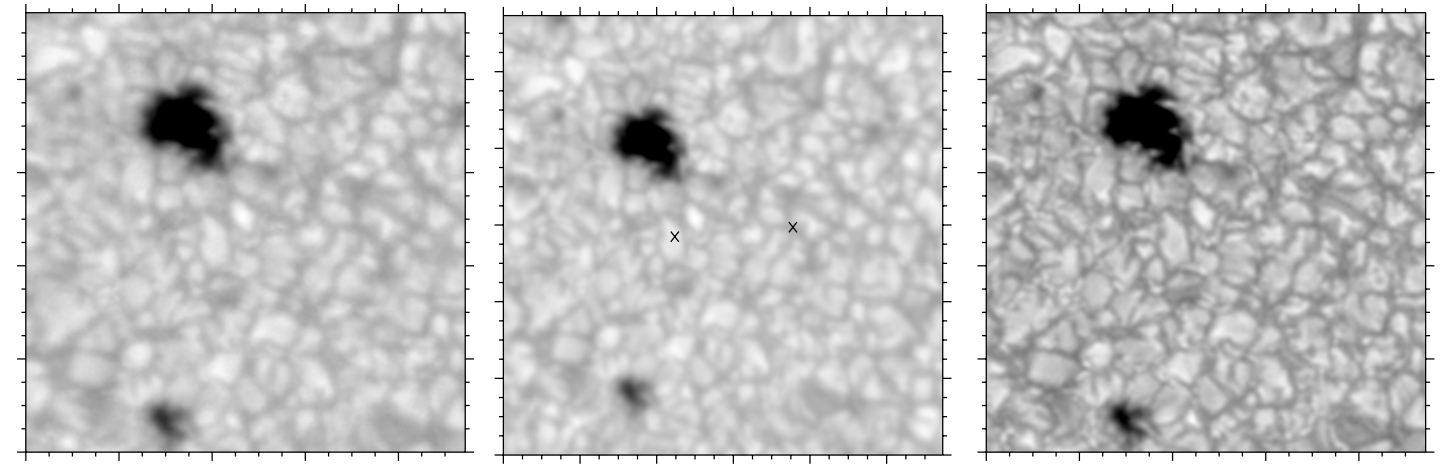

Fig. 2. Left: best broad-band image taken from first scan. Middle: averaged broad-band image (over 76 images). The black crosses indicate the regions discussed in Sect. 4.2. Right: broad-band speckle reconstruction. One minor tick mark corresponds to one arcsec.

For the present work, we adopted a different approach: we performed a spectral sampling with equidistant steps of $0.84 \mathrm{pm}$ and used five adjacent wavelength positions to perform the deconvolution. For the data presented here, this averaging procedure has very little effect on reconstructed line profiles.

The following two subsections describe in more detail the deconvolution process and the reconstructed images.

\subsection{Broad-band speckle reconstruction}

The broad-band TESOS images and the G-band images were speckle reconstructed using the Kiepenheuer Institute Speckle Imaging Package (KISIP) developed by O. von der Lühe and modified by K. Mikurda, J. Setiawan and F. Wöger. The code uses an extended Knox-Thompson algorithm (Mikurda et al. 2003) to reconstruct the object phases while the object amplitudes are recovered utilizing Labeyrie's technique (Labeyrie 1970). The code itself and its performance will be described in detail in a forthcoming publication (Mikurda \& von der Lühe 2006).

Figure 2 shows a typical result of the speckle reconstruction in the broad-band channel of TESOS. Displayed are the best broad-band image (left, 5.3\% rms intensity contrast), the scanaveraged broad-band image (middle, $4.7 \% \mathrm{rms}$ contrast), and the speckle reconstruction (right, $7.0 \% \mathrm{rms}$ contrast). On average, the broad-band speckle reconstruction leads to $40 \%$ higher intensity contrasts. The speckle reconstructed G-band images show rms intensity contrasts of $9.3 \%$.

The fact that the speckle technique is applied to already seeing-corrected data accomplished by the adaptive optic system deserves a comment. The speckle technique makes assumptions about the statistics of the wavefront aberrations introduced by the turbulent Earth's atmosphere, in KISIP we adopted Korff's model of the Speckle Transfer Function (STF) (Korff 1973).

The dynamic aberrations of a wavefront are compensated by the $\mathrm{AO}$ system with an efficiency lower than one, so that residual wavefront aberrations are present. The statistics of the residual wavefront errors are different from the error statistics of an uncompensated wave, but the effects on the STF are, to a first order approximation, similar to that of an uncompensated wave with improved seeing (Cagigal \& Canales 2000a,b).

The AO system also loses efficiency with increasing distance from the lock point, but a field dependent STF corrects to first order the anisoplanatism.

This issue has been addressed in more detail in Mikurda \& von der Lühe (2006).

\subsection{Narrow-band deconvolution}

Following the approach of Keller \& von der Lühe (1992) and Krieg et al. (1999) the narrow-band images were reconstructed from the following expression:

$\bar{O}_{j}^{\mathrm{n}}=\frac{\left\langle I_{j}^{\mathrm{n}}\left(I_{j}^{\mathrm{b}}\right)^{*}\right\rangle}{\left\langle\left|I_{j}^{\mathrm{b}}\right|^{2}\right\rangle+\left\langle\left|N_{j}^{\mathrm{b}}\right|^{2}\right\rangle} \bar{O}^{\mathrm{b}}$,

where $*$ is the complex conjugate, and $O^{\mathrm{b}}, I_{j}^{\mathrm{n}}, I_{j}^{\mathrm{b}}$ denote, in Fourier domain, the speckle-reconstructed broad-band image and the instantaneous narrow- and broad-band images, respectively. The index $j(j=0, \ldots, 75)$ is introduced to distinguish between individual frames in the broad-band channel and thus different atmospheric realizations and also to label different wavelength positions in the narrow-band channel.

For the data presented here an averaging process, $\langle\ldots\rangle$, over five narrow-band filtergrams $(j=j-2, \ldots, j+2)$ is performed. $\left\langle\left|N_{j}^{\mathrm{b}}\right|^{2}\right\rangle$ is the average noise level as deduced from the broad-band images. We assume a frequency independent ("white") noise spectrum. Averaging is done over frequencies that are beyond the telescope cut-off frequency.

Equation (1) is valid only within an isoplanatic patch, but the considered field of view is much larger. The solution here is to divide a field of view into several subfield, perform the reconstruction in each subfield and then recombine them. The sub-fielding procedure used here is described in depth by von der Lühe (1993).

In order to allow for a fair comparison between the data before and after the restoration process, the un-restored data cube (76 wavelength points) has been reduced to the same number of wavelength points as the restored data cube (71 wavelength points). Therefore, a box-car average over five images is performed to the un-restored data cube.

The result of the deconvolution process is demonstrated in Fig. 3 (lower panel) based on the restoration of the first scan of our observations. For comparison, the un-restored but averaged corresponding filtergrams are shown in the upper panel. Displayed are five filtergrams at different wavelength positions during the scan. In the right upper corner we display a mean quiet sun profile versus frame number to visualize the relative position of the filtergrams (vertical dotted lines). In the narrow-band continuum, the deconvolution technique is almost as efficient as the broad-band speckle reconstruction, where we achieve an average contrast enhancement of $30 \%$. We expect that the deconvolution algorithm works equally well through the line profile, because the procedure remains the same. Direct visual 

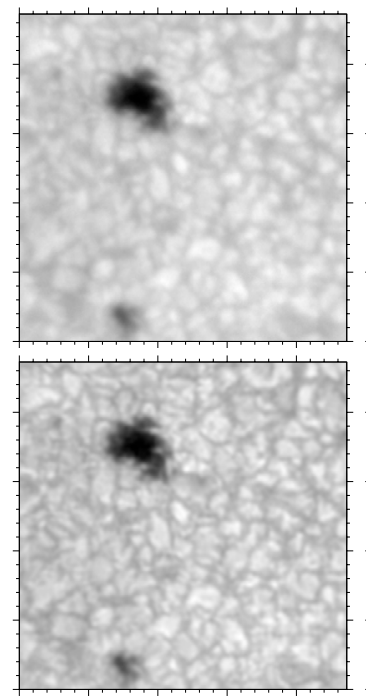
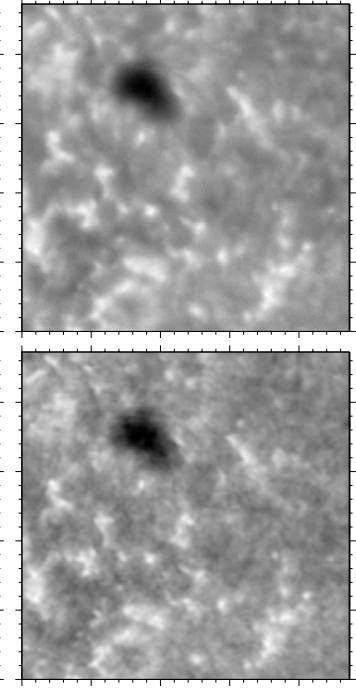
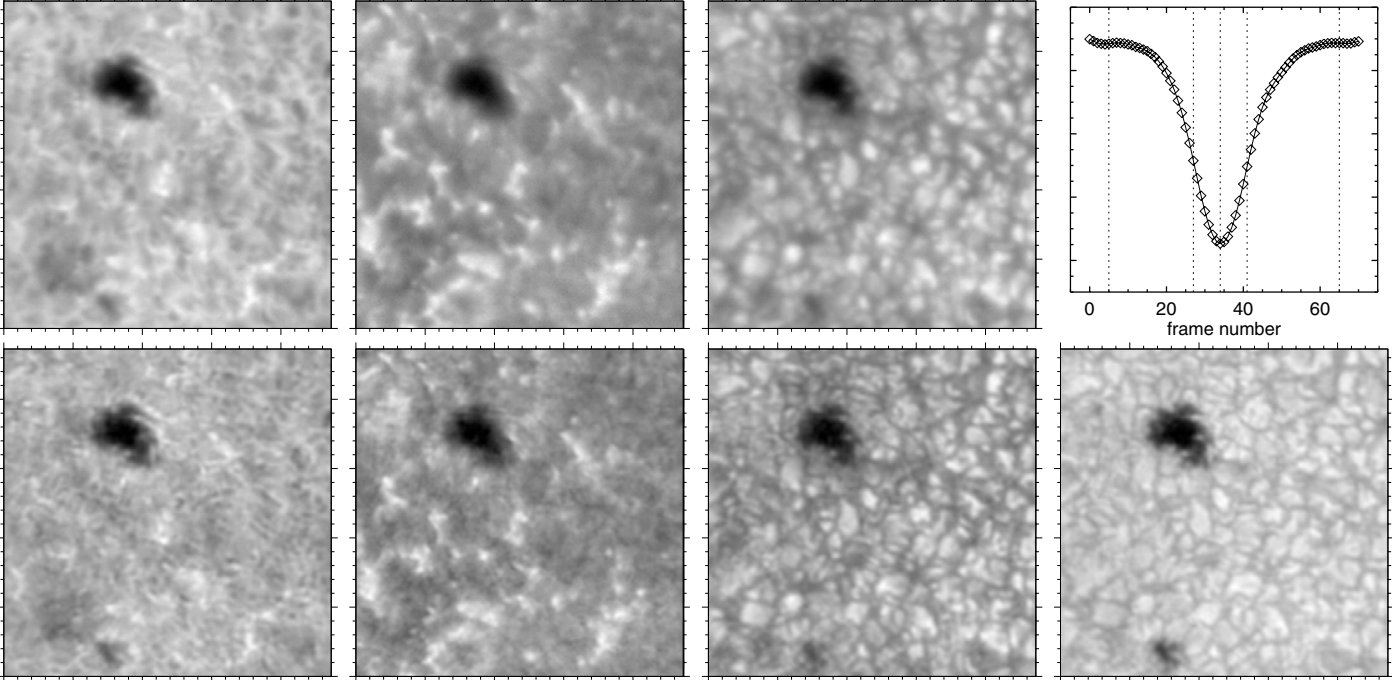

Fig. 3. Upper row: narrow-band filtergrams before reconstruction. Right panel: mean quiet sun profiles versus frame number, vertical axis 0.1 to 1.2 $\cdot I_{\text {cont }}$. Vertical dotted lines indicate the wavelength positions of the displayed filtergrams. Lower row: narrow-band filtergrams after the restoration process. Left to right: blue continuum (frame number 5), blue line wing (27), line core (34), red line wing (41), and red continuum (65). One minor tick mark corresponds to one arcsec. All images are scaled individually.
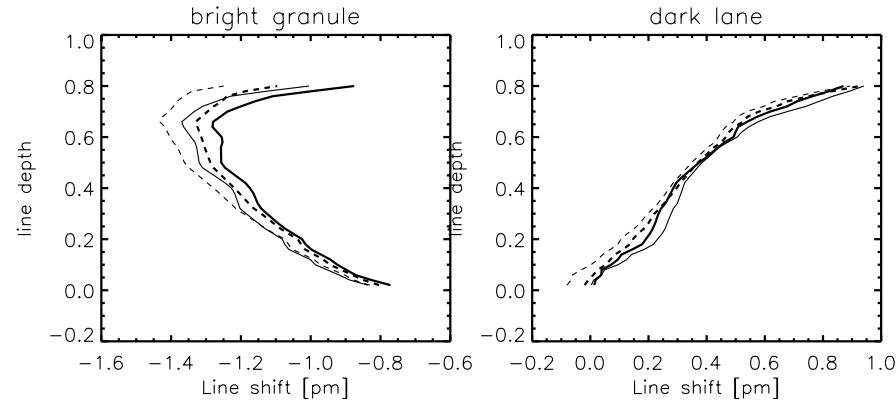

Fig. 4. Bisectors before (dashed) and after (solid) the deconvolution process for locations shown in Fig. 2 (middle). Thin lines: bisectors averaged over four pixels. Thick lines: bisectors averaged over nine pixels.

inspection of the filtergrams in Fig. 3 reveals the presence of a weak small-scale grainy pattern in the deconvolved filtergrams. The pattern is more pronounced in the line wing and line core and less obvious in the continuum but still present. The spatial scale of this pattern is close to the resolution limit, the grains span 2-3 pixel which corresponds to $0.18-0.27$ arcsec. Such a pattern has been observed before in narrow-band image reconstructed data (see e.g. Krieg et al. 1999). The pattern is most likely band-pass limited noise caused by the lack of sufficient signal-to-noise ratio at spatial frequencies close to the diffraction limit. This effect is getting more severe in the line wing and line core filtergrams than in the continuum. Van Noort et al. (2005) observed a similar effect in the magnetograms reconstructed with the phase-diversity technique and concluded that it can be reduced by taking more frames at each wavelength position.

The question appears, if the existence of the grainy pattern has any influence on the bisectors of single structures. In order to check that, we analyzed several line profiles and the corresponding bisectors (cf. Sect. 5) of individual structures. In Fig. 4 we display two different bisectors (for a bright granule and an intergranular lane as indicated with crosses in Fig. 2) before (dashed) and after the deconvolution (solid). The bisectors are averaged over four (thin lines) and nine pixels (thick lines), respectively. First of all, one can see that there are no significant differences in the bisectors before and after the deconvolution process. Moreover, increasing the averaging area does not significantly change the result. Therefore, we conclude that the presence of the grainy pattern in general does not alter the spectral information.

The filtergrams taken in the blue wing of the spectral line are quite different from those obtained in the red wing at the same line depression (compare 27 and 41 in Fig. 3). The different appearance of the filtergrams is a natural consequence of the interaction between convection and radiation. This velocity-intensity correlation for an another photospheric spectral line (BaII $455.4 \mathrm{~nm}$ ) is described in detail in (Sütterlin et al. 2001).

As a test, we shifted the position of the spectral line to a common position at every pixel. The result is illustrated in Fig. 5 for the deconvolved filtergrams. The large differences between the blue and the left wing are virtually gone. Residual differences are due to line asymmetries which we did not account for.

In a spectral line this situation is further complicated by the fact that the $\tau=1$ surface is influenced by both thermal and Doppler effects causing a wide range of line shifts, line widths and line asymmetries (e.g. Stein \& Nordlund 1998; Asplund et al. 2000). In general the temperature gradient above granules appears to be steeper giving rise to stronger and narrower line profiles that are blue shifted due to upwelling convective motions. The temperature gradient above intergranular lanes tends to be shallower leading to weaker and broader line profiles that are red shifted due to the downflows in the intergranular lanes.

\section{Spectral analysis}

The pre-processed and reconstructed data cube leaves us with a line profile sampled at 71 wavelength points at each spatial position (pixel). As already mentioned before, the un-restored data cube is prepared in the sense that an averaging process in spectral direction similar to that performed during the deconvolution is applied in order to allow for a direct comparison between the data before and after the restoration process. A line 

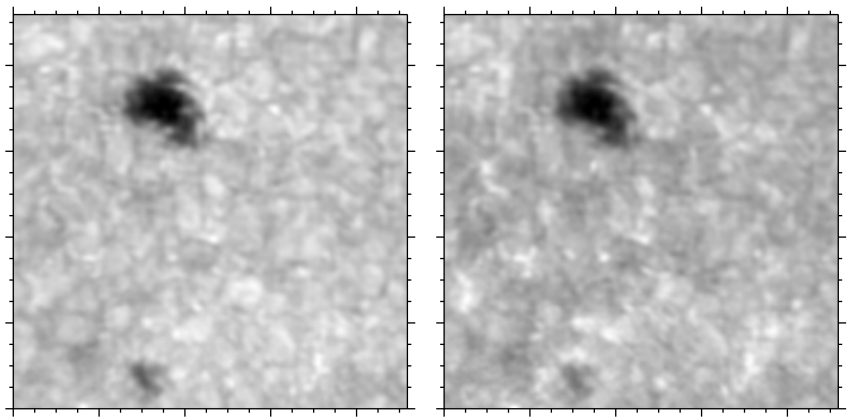

Fig. 5. Reconstructed filtergrams taken from the blue (left panel) and red wing of the line profile. Prior to the image reconstruction, the spectral line was shifted to a common position at each pixel. One major tick mark corresponds to five arcsecs.
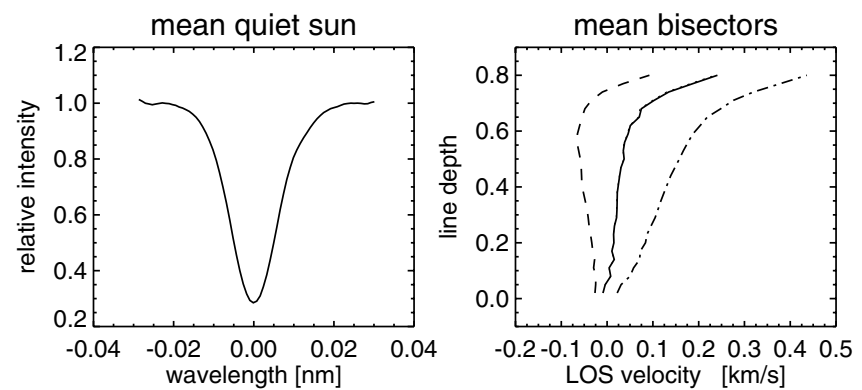

Fig. 6. Left: spatially and temporally averaged line profile of the solar granulation inside the TESOS FOV. Right: corresponding bisector (solid). Dashed: quiet sun granulation. Dashed-dotted: abnormal granulation. The profile and the bisectors are derived from the deconvolved data set.

bisector analysis is performed at each spatial position. For each line profile, 30 bisector positions are calculated at intensity levels between $2 \%$ and $80 \%$ of the line depression. Line depths of 0.0 and 1.0 correspond to the line center and the continuum, respectively. Bisector levels larger than $80 \%$ are not taken into account since the bisector positions in the extreme line wings are very sensitive to noise. The bisectors are used to determine (a) the line-core Doppler shift by averaging the bisector positions between the $2 \%$ and $20 \%$ level of the line depression and (b) the line-wing Doppler shift by averaging between $60 \%$ and $80 \%$ of the line depression (Tritschler et al. 2004). Positive (dark) velocities point away from the observer and correspond to red-shifts, and negative (bright) velocities correspond to blue-shifts. The line core position of the granulation (averaged over the FOV) serves as velocity reference.

\subsection{Line profiles}

We construct binary masks based on the broad-band reconstruction by setting intensity threshold values to differ between and identify special regions of interest: quiet sun, granules, intergranular lanes, inside part and edge of the pore. The G-band bright points are identified by using the G-band speckle reconstruction. The masks are subsequently used to extract individual (G-band) and compute spatially and/or temporally averaged line profiles.

Figure 6 displays the spatially and temporally averaged line profile (right) and the corresponding bisector (left) of the solar granulation inside the TESOS FOV after the restoration process. Differences between the profiles and bisectors before and after the restoration are negligible, which is ascribed to the fact that spatially (and temporally) averaged intensities should be conserved by the reconstruction technique. As is obvious from the narrow-band continuum filtergrams shown in Fig. 3 (e.g. left, upper panel), the granular pattern located on the center side (toward the active region) shows much less contrast and seems spatially distorted ("abnormal" granulation, see e.g. Dunn \& Zirker 1973) if compared to the granulation on the limb side (away from the active region). The division of the FOV into two halves already reveals the intrinsic differences between the two areas, particularly if looking at the corresponding mean bisectors (normal: dashed, abnormal: dashed-dotted) as shown in Fig. 6. The mean bisector of the abnormal granulation is inclined toward the red, indicating a continuous acceleration toward the surface.

The mean bisector of the rather undisturbed granulation is almost vertical with a slight asymmetry toward the blue showing the expected C-shape (Dravins et al. 1981; Dravins 1982), though less pronounced than from observations far outside active regions.

In Fig. 7 we display spatially and temporally averaged profiles (upper row) and the corresponding mean bisectors (middle row) for selected regions of interest: the brightest granules, the darkest intergranular lanes, the darkest parts of the pore, and the edge of the pore. Also shown are the spatially averaged bisectors extracted from the individual scans (lower row). To demonstrate the effect of the reconstruction process, the corresponding data before (dotted) and after the deconvolution (solid) are plotted. The line profiles are normalized with respect to the local continuum intensity of the averaged granulation, determined from the reconstructed data (see Fig. 6). The bisectors are calibrated with respect to the line core position of the spatially and temporally averaged quiet sun profile (whole FOV).

Our results are essentially consistent with those from onedimensional spectroscopic data (e.g. Hanslmeier et al. 1991b). The advantage of 2D spectroscopy is the possibility to clearly distinguish between bright granules and intergranular lanes. Our results concerning the bisectors shape in "quiet" and "abnormal" granulation agree with previous findings for active and non- active regions (e.g. Hanslmeier et al. 1991a).

\subsubsection{Bright granules and intergranular lanes}

The reconstructed mean line profile for the brightest granules in the FOV (Fig. 7, upper panel, left) shows an enhanced continuum intensity, narrower wings, and a slightly higher line core intensity. For the darkest intergranular lanes, the deconvolution leads to a decrease of the continuum intensity, broader wings, and a slightly lower line core intensity. Thus, bright structures become brighter and dark structures become darker by the deconvolution process, as expected.

The close correspondence of the of bisector shapes before and after the deconvolution proves that line asymmetries are not altered but preserved (middle and bottom row). Differences in the bisector shape are restricted to the (far) wing closer to the continuum intensity level reflecting the broadening and narrowing of the line profiles due to the deconvolution. The individual granular and intergranular bisectors (bottom row) cover a time period of only $2.5 \mathrm{~min}$ and we thus do not expect to see any large variations.

We follow the aforementioned approach and divide the FOV into two parts representative for quiet sun (dashed) and abnormal conditions (solid thick). The mean bisectors averaged over the brightest structures in the normal half show the expected blue asymmetry or reversal of the bisector in deep photospheric 

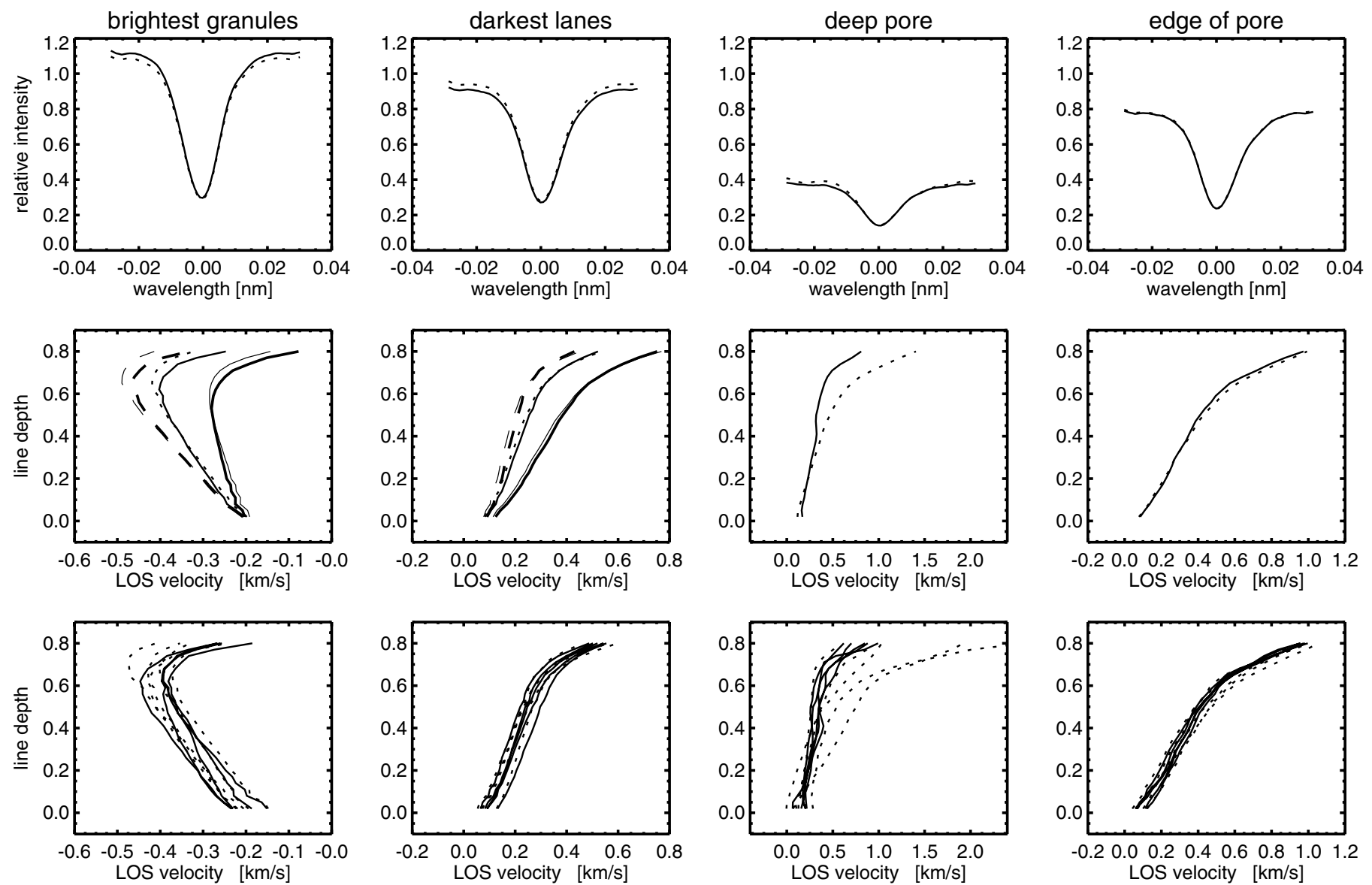

Fig. 7. Averaged line profiles and bisectors before (dotted) and after (solid) the deconvolution process for selected regions of interest. Top row: spatially and temporally (over five scans) averaged line profiles. Middle row: like top but corresponding bisectors. Dashed lines: normal granulation. Thick solid lines: abnormal granulation. Bottom row: spatially averaged bisectors for the five scans.

layers where a maximum LOS-velocity of $-0.5 \mathrm{~km} \mathrm{~s}^{-1}$ is reached. This blue asymmetry is significantly reduced in the abnormal area throughout the height range spanned by the spectral line. The bisector is less inclined (almost vertical) and shows a less pronounced reversal. Furthermore, the abnormal intergranular lanes lead on average to more inclined bisectors with a strong red asymmetry $\left(0.6-0.7 \mathrm{~km} \mathrm{~s}^{-1}\right)$ compared to the normal intergranular lanes $\left(0.2 \mathrm{~km} \mathrm{~s}^{-1}\right)$.

\subsubsection{Pore and edge of pore}

The spatially and temporally averaged line profiles of the darkest part of the pore and the edge of the pore show basically the same tendency than that derived from the darkest intergranular lanes before and after the deconvolution: continuum intensities are decreased and the lines are broader. There is a close match for the edge of the pore before and after the reconstruction. The bisectors are strongly inclined toward the red (up to $1 \mathrm{~km} \mathrm{~s}^{-1}$ ) giving rise for the presence of a downflow region in deep photospheric layers around the pore. These downflows have been observed before (e.g. Keil et al. 1999; Sankarasubramanian \& Rimmele 2003; Rimmele 2004) and are theoretically predicted (e.g. Pizzo 1986). The numerical simulations of Steiner et al. (1998) indicate the presence of strong and narrow downflow jets that border a magnetic flux sheet. These flows are fed by horizontal flows in the upper layers and driven by radiative cooling of the plasma surrounding the flux sheet. Although the simulations are for magnetic elements, similar processes are conceivable to take place at the border of larger magnetic structures like pores.

However, the mean bisectors for the pore differ considerably before and after the reconstruction, in particular for bisector levels close to the continuum. Taking a closer look at the individual spatially averaged bisectors that contribute to the mean bisector, we find that in particular two bisectors resulting from the second and third scan lead to the large deviation. Inspecting individual line profiles inside the pore from these two scans reveal the existence of a highly red-shifted line satellite. A possible explanation for this is the presence of a strong downflow inside the pore. However, in the far red wing the line is blended with two molecular lines which might cause or influence the observed asymmetry: $\mathrm{MgH}$ at $557.628 \mathrm{~nm}$ and $\mathrm{C}_{2}$ at $557.636 \mathrm{~nm}$. The $\mathrm{MgH}$ line only shows up at low temperatures because the molecule has a very low dissociation energy of $1.3 \mathrm{eV}$. The $\mathrm{C}_{2}$ line disappears at lower temperatures (high dissociation energy of $6.2 \mathrm{eV}$ ) because it comes from an excited state that only gets populated at higher temperatures (priv. comm. Han Uitenbroek). This line flag will be subject of a separate investigation.

\subsubsection{Isolated G-band bright point}

The proper motion of G-band bright points of (GBPs) is already well understood (e.g. Berger et al. 1998; Nisenson et al. 2003), but high-resolution measurements of line-of-sight velocities are 


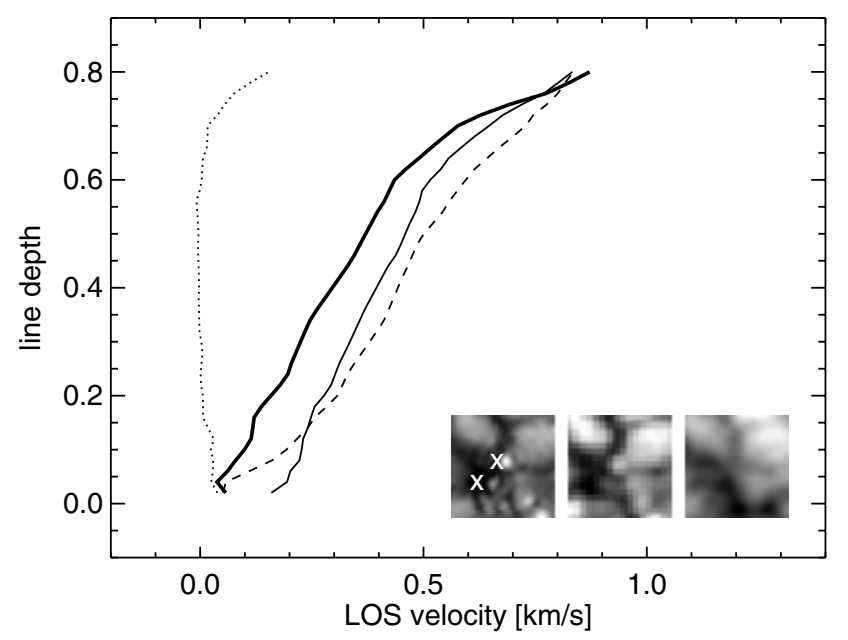

Fig. 8. Bisectors of an isolated G-band bright point and its surrounding. Thick solid line: G-band bright point. Solid line: immediate surrounding of GBP (upper cross on the inlay). Dashed line: intergranular lane at 0.6 arcsec distance (lower cross on the inlay). Dotted line: bright granule at 0.5 arcsec distance. The inlay shows the analyzed GBP and its surroundings (left to right): G-band image, broad-band continuum image, line-wing velocity. The FOV is 2.2 arcsec.

scarce. Many GBPs seem to appear in very compact "groups" or "chains" (e.g. Berger et al. 1995; Berger \& Title 2001) in the intergranular lanes. In order to allow for a comparison with the surrounding intergranular area, we chose an isolated GBP belonging to the abnormal granulation region for our further analysis. The bisectors of the investigated GBP and its surroundings are shown in Fig. 8.

The investigated bright point is associated with a downflow (in agreement with e.g. Langhans et al. 2004), but it does not show any line-of-sight motion relative to the immediate intergranular surroundings. The bisector of the GBP is quite similar to that of the surrounding intergranular plasma, i.e. the velocity gradient is about the same inside and outside the bright point. Scattered light can hardly explain our result: the line asymmetry of the nearby granulation is quite different, and scattering from the dark intergranular area to the bright point is certainly insufficient.

Note that Beck et al. (2006) find a strong downflow of the material inside a bright point that is just being formed in agreement with 2D numerical simulations (Steiner et al. 1998). In recent 3D MHD simulations the vertical velocity at the $\tau=1$ level (Shelyag et al. 2004, Fig. 1) does not show large differences between the flows inside magnetic flux concentrations and those of intergranular lanes. However, some flux concentrations do have a downflow or upflow relative to their immediate surroundings.

Our result and that of Beck et al. (2006) may be explained and brought together by studying the life history of bright points. This will be addressed in a detailed study of bright-point development which is beyond the scope of this paper.

\subsection{Line-of-sight velocities}

As a further example for the physical quantities deduced from the 2D-spectra, Fig. 9 shows maps of the line-of-sight velocity measured from Doppler shifts in the line wing (left) and the line core (right) before (upper panels) and after (lower panels) the restoration process. The Dopplergrams show the well-known difference between quiet and abnormal granulation

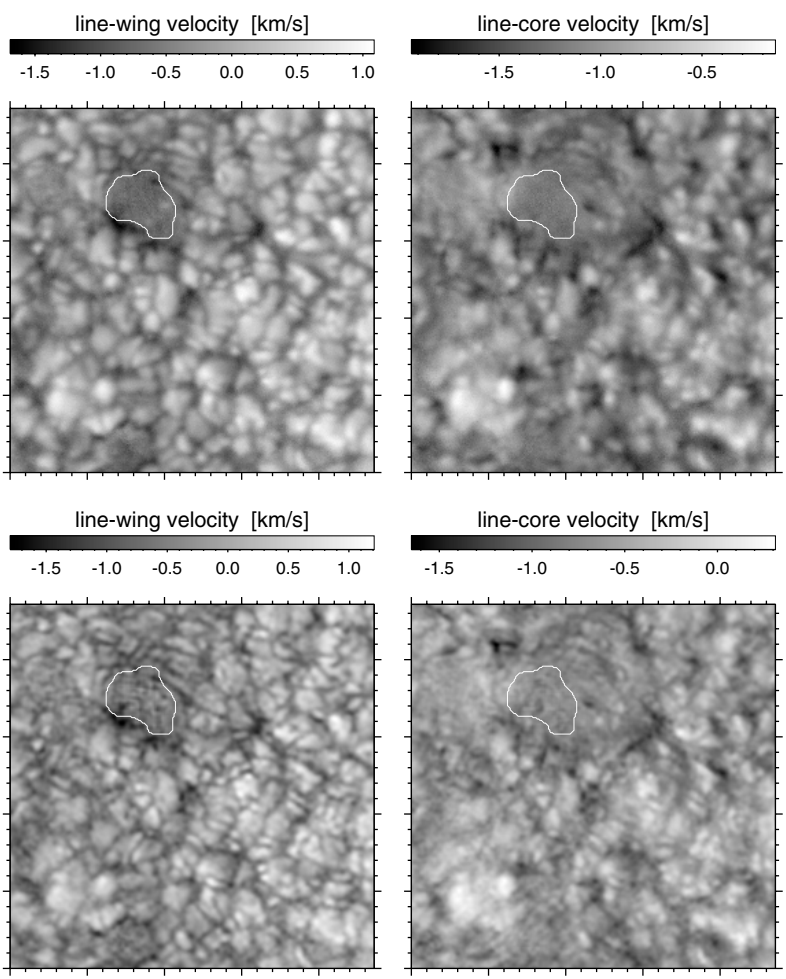

Fig. 9. Dopplergrams deduced from red line wing and the line core intensities. Upper panel: unrestored data. Lower panel: deconvolved data.

(Title et al. 1992): the velocity measured from the line wing is less pronounced in the disturbed region compared to the quiet sun and the velocity amplitudes are smaller. No such difference is visible in the line-core velocity (right panels of Fig. 9). Note the narrow downflow area (dark) around the pore near the bottom of the ling-wing Dopplergrams. In higher layers, i.e. in the linecore data, only the strongest up-and downflows have survived. The weak grainy pattern in the restored data is best seen in areas with a low signal-to-noise ratio (e.g. inside the pores). A similar effect can be seen in the velocity maps presented for example by Hirzberger \& Kneer (2001) and calculated from deconvolved 2D spectral data. More surprisingly, a similar pattern occurs in the Dopplergrams shown by Sütterlin et al. (2001) and obtained from five speckle-reconstructed filtergrams. This fact suggests that the artifacts in the Doppler maps are not the intrinsic property of the technique discussed here, but - as already mentioned - are the effect of low signal-to-noise ratio in certain areas of the image. The pattern distribution seems to be random and the averaged bisector from the reconstructed data matches the original one very well in most cases (see Fig. 7). The rms-value of the pattern is about $100 \mathrm{~m} / \mathrm{s}$ in the line core and about $160 \mathrm{~m} / \mathrm{s}$ in the line wing.

\section{Conclusions}

We have applied image-reconstruction and deconvolution techniques to high-resolution spectroscopic data. We performed a detailed spectral analysis of a short sequence of $2 \mathrm{D}$-spectra of solar granulation including two pores. This analysis included the measurement of line position and depth, as well as the line asymmetry. The latter quantity is characterized in form of line bisectors. We compared quiet and disturbed (abnormal) granulation, measured the height dependence of the line shift separately for 
bright granules, dark intergranular lanes and an isolated bright point. The main results are:

- The disturbed granulation shows a more diffuse velocity pattern with smaller amplitudes if compared to the quiet sun, in agreement with previous findings.

- There is a significant difference between the line asymmetry in quiet and disturbed granulation: the line bisector in the disturbed dark lanes is much more symmetric than in the quiet area, at least in the higher layers. The contrary behavior is seen in the bright granules.

- The LOS velocity and the line asymmetry of the isolated GBP after the formation phase do not differ from those observed in the intergranular lanes.

Based on the scientific results, we investigated, if and how the reconstruction process modifies the photometric properties of our data. The main findings concerning the photometric quality of the reconstructed spectra are summarized as follows:

- Average intensities are preserved and local changes on small spatial scales have the correct sign: bright granules become brighter and dark lanes become darker through the reconstruction.

- Line profiles of bright structures show a decreased line width and those of dark structures have an increased line width.

- Line asymmetries are not altered, i.e. the shape of the line bisectors is preserved.

- There is a weak grainy pattern in the reconstructed data, especially in areas of low signal-to noise ratio.

We have shown that the image reconstruction process does not introduce artefacts to the line profiles, so they can be used for the quantitative spectroscopy. In contrast to 1D spectroscopy, the temporal resolution of $2 \mathrm{D}$-spectroscopic data cannot easily be increased at the cost of area coverage. The existence of a weak grainy pattern in many realizations of speckle-reconstructed Doppler maps may point to an intrinsic limitation of 2D spectroscopy. The solar photon flux per resolution element is constant, i.e. independent of the telescope used, hence the product of temporal, spatial and spectral resolution defines the signal-tonoise ratio. In order to obtain a certain data quality, trade-offs between these parameters may be needed.

Acknowledgements. The German Vacuum Tower Telescope on Tenerife, Canary Islands, is operated by the Kiepenheuer Institut für Sonnenphysik, Freiburg, Germany. Part of this work (KM) was supported by the DFG under grant Schm $1168 / 3$. Part of this work (AT) was supported by NSF under grants ATM 0086999 and AST MRI 00-79482, and by NASA under grant NAG 5-12782.

\section{References}

Asplund, M., Nordlund, Å., Trampedach, R., et al. 2000, A\&A, 359, 729 Beck, C., Schmidt, W., Bellot Rubio, L. R., et al. 2006, in ASP Conf. Ser. Proceedings of the 4th Solar Polarization Workshop

Berger, T. E., \& Title, A. M. 2001, ApJ, 553, 449

Berger, T. E., Schrijver, C. J., Shine, R. A., et al. 1995, ApJ, 454, 531

Berger, T. E., Loefdahl, M. G., Shine, R. S., \& Title, A. M. 1998, ApJ, 495, 973

Cagigal, M. P., \& Canales, V. F. 2000a, Opt. Soc. Am. J. A, 17, 903

Cagigal, M. P., \& Canales, V. F. 2000b, Opt. Soc. Am. J. A, 17, 1312

de Boer, C. R., \& Kneer, F. 1992, A\&A, 264, L24
Dravins, D. 1982, ARA\&A, 20, 61

Dravins, D., Lindegren, L., \& Nordlund, A. 1981, A\&A, 96, 345

Dunn, R. B., \& Zirker, J. B. 1973, Sol. Phys., 33, 281

Gonsalves, R. A., \& Chidlaw, R. 1979, in Applications of digital image processing III; Proceedings of the Seminar, San Diego, Calif., August 2729, 1979 (A80-39704 16-35) Bellingham, Wash., Society of Photo-Optical Instrumentation Engineers. Research supported by the Hughes Aircraft Co. and U.S. Air Force, 32

Hanslmeier, A., Mattig, W., \& Nesis, A. 1991a, A\&A, 244, 521

Hanslmeier, A., Mattig, W., \& Nesis, A. 1991b, A\&A, 251, 669

Hirzberger, J., \& Kneer, F. 2001, A\&A, 378, 1078

Keil, S. L., Balasubramaniam, K. S., Smaldone, L. A., \& Reger, B. 1999, ApJ, 510,422

Keller, C. U., \& Johannesson, A. 1995, A\&AS, 110, 565

Keller, C. U., \& von der Lühe, O. 1992, A\&A, 261, 321

Keller, C. U., Plymate, C., \& Ammons, S. M. 2003, in Innovative Telescopes and Instrumentation for Solar Astrophysics, ed. S. L. Keil, \& S. V. Avakyan, Proc. SPIE, 4853, 351

Kentischer, T. J., Schmidt, W., Sigwarth, M., \& Uexkuell, M. V. 1998, A\&A, 340,569

Knox, K. T., \& Thompson, B. J. 1974, ApJ, 193, L45

Korff, D. 1973, Opt. Soc. Amer. J., 63, 971

Krieg, J., Wunnenberg, M., Kneer, F., Koschinsky, M., \& Ritter, C. 1999, A\&A, 343,983

Löfdahl, M. G., \& Scharmer, G. B. 1994, in Image Reconstruction and Restoration, T. J. Schulz, \& D. L. Snyder, Proc. SPIE, 2302, 254

Löfdahl, M. G., \& Scharmer, G. B. 2003, in Innovative Telescopes and Instrumentation for Solar Astrophysics, ed. S. L. Keil, \& S. V. Avakyan, Proc. SPIE, 4853, 567

Labeyrie, A. 1970, A\&A, 6, 85

Langhans, K., Schmidt, W., \& Rimmele, T. 2004, A\&A, 423, 1147

Mikurda, K., \& von der Lühe, O. 2006, Sol. Phys., in press

Mikurda, K., von der Lühe, O., \& Wöger, F. 2003, Astron. Nachr. Suppl., 324, 112

Nisenson, P., van Ballegooijen, A. A., de Wijn, A. G., \& Sütterlin, P. 2003, ApJ, 587,458

Paxman, R. G., \& Fienup, J. R. 1988, Opt. Soc. Amer. J., 5, 914

Pizzo, V. J. 1986, ApJ, 302, 785

Rimmele, T. R. 2004, ApJ, 604, 906

Rimmele, T. R., Richards, K., Hegwer, S., et al. 2004, in Telescopes and Instrumentation for Solar Astrophysics, ed. S. Fineschi, \& M. A. Gummin, Proc. SPIE, 5171, 179

Rouppe van der Voort, L. H. M., Löfdahl, M. G., Kiselman, D., \& Scharmer, G. B. 2004, A\&A, 414, 717

Sütterlin, P., \& Wiehr, E. 2000, Sol. Phys., 194, 35

Sankarasubramanian, K., \& Rimmele, T. 2003, ApJ, 598, 689

Scharmer, G. B., Dettori, P. M., Lofdahl, M. G., \& Shand, M. 2003, in Innovative

Telescopes and Instrumentation for Solar Astrophysics, ed. S. L. Keil, \& S. V. Avakyan, Proc. SPIE, 4853, 370

Shelyag, S., Schüssler, M., Solanki, S. K., Berdyugina, S. V., \& Vögler, A. 2004, A\&A, 427, 335

Starck, J. L., Pantin, E., \& Murtagh, F. 2002, PASP, 114, 1051

Stein, R. F., \& Nordlund, A. 1998, ApJ, 499, 914

Steiner, O., Grossmann-Doerth, U., Knoelker, M., \& Schuessler, M. 1998, ApJ, 495, 468

Sütterlin, P., Rutten, R. J., \& Skomorovsky, V. I. 2001, A\&A, 378, 251

Title, A. M., Topka, K. P., Tarbell, T. D., et al. 1992, ApJ, 393, 782

Tritschler, A., Schmidt, W., Langhans, K., \& Kentischer, T. 2002, Sol. Phys., 211,17

Tritschler, A., Schlichenmaier, R., Bellot Rubio, L. R., et al. 2004, A\&A, 415, 717

van Noort, M., van der Voort, L., \& Löfdahl, M. 2005, Sol. Phys., in press

von der Lühe, O. 1993, A\&A, 268, 374

von der Lühe, O., Widener, A. L., Rimmele, T., Spence, G., \& Dunn, R. B. 1989, A\&A, 224, 351

von der Lühe, O., Soltau, D., Berkefeld, T., \& Schelenz, T. 2003, in Innovative

Telescopes and Instrumentation for Solar Astrophysics, Proc. SPIE, ed. S. L.

Keil, \& S. V. Avakyan, 4853, 187

Weigelt, G. P. 1977, Opt. Comm., 21, 55 